\title{
Terminal complement complex formation is associated with intervertebral disc degeneration
}

\author{
Graciosa Q. Teixeira ${ }^{1}$ (1) $\cdot$ Zhiyao Yong $^{1} \cdot$ Raquel M. Goncalves ${ }^{1,2,3,4} \cdot$ Amelie Kuhn $^{5} \cdot$ Jana Riegger $^{5} \cdot$ Helena Brisby $^{6,7}$. \\ Helena Barreto Henriksson ${ }^{6,7,8}$. Michael Ruf ${ }^{9} \cdot$ Andreas Nerlich $^{10}$. Uwe M. Mauer ${ }^{11}$ • Anita Ignatius ${ }^{1}$. \\ Rolf E. Brenner ${ }^{5}$. Cornelia Neidlinger-Wilke ${ }^{1}$ (D)
}

Received: 1 April 2020 / Revised: 16 June 2020 / Accepted: 3 September 2020 / Published online: 16 September 2020

(c) The Author(s) 2020

\begin{abstract}
Purpose The complement system is a crucial part of innate immunity. Recent work demonstrated an unexpected contribution to tissue homeostasis and degeneration. This study investigated for the first time, in human disc tissues, the deposition profile of the complement activation product terminal complement complex (TCC), an inflammatory trigger and inducer of cell lysis, and its inhibitor CD59, and their correlation with the degree of disc degeneration (DD).

Methods Disc biopsies were collected from patients diagnosed with DD $(n=39$, age $63 \pm 12)$ and adolescent idiopathic scoliosis (AIS, $n=10$, age $17 \pm 4)$ and compared with discs from healthy Young $(n=11$, age $7 \pm 7)$ and Elder $(n=10$, age $65 \pm 15$ ) donors. Immunohistochemical detection of TCC and CD59 in nucleus pulposus (NP), annulus fibrosus (AF) and endplate (EP) was correlated with age, Pfirrmann grade and Modic changes.

Results Higher percentage of TCC + cells was detected in the NP and EP of DD compared to Elder $(P<0.05)$, and in the EP of Young versus Elder $(P<0.001)$. In DD, TCC deposition was positively correlated with Pfirmann grade, but not with Modic changes, whereas for Young donors, a negative correlation was found with age, indicating TCC's involvement not only in DD, but also in early stages of skeletal development. Higher CD59 positivity was found in AIS and DD groups compared to Young $(P<0.05)$, and it was negatively correlated with the age of the patients.

Conclusion TCC deposition positively correlated with the degree of disc degeneration. A functional relevance of TCC may exist in DD, representing a potential target for new therapeutics.
\end{abstract}

Keywords Back pain · Innate immunity $\cdot$ Scoliosis $\cdot$ Inflammation $\cdot$ Complement system

\section{Introduction}

Disc degeneration (DD) and associated inflammation often lead to low back pain, one of the major causes of disability worldwide, representing a very large economic burden [1]. Current treatments (physiotherapy, disc replacement, among others) decrease symptoms progression, but fail to restore the native intervertebral disc (IVD) properties. The highest risk factors of early disc pathologies include mechanical overloading, genetic predisposition and/or smoking [2].

Electronic supplementary material The online version of this article (https://doi.org/10.1007/s00586-020-06592-4) contains supplementary material, which is available to authorized users.

Cornelia Neidlinger-Wilke

cornelia.neidlinger-wilke@uni-ulm.de

Extended author information available on the last page of the article
Characteristic features of degenerated IVDs are structural changes, such as reduced disc height, a transformation of the central gelatinous nucleus pulposus (NP) into a more fibrotic tissue, with reduced water binding capacity due to progressing degradation and less proteoglycan production. The annulus fibrosus (AF) also suffers increased breakdown of the structural matrix components which contribute to fissures and tears $[3,4]$.

Understanding the mechanisms leading to the imbalance of disc homeostasis is subject of recent investigations. It is known that a reduced permeability of the adjacent cartilaginous endplates (EP) for nutrients and metabolites leads to enhanced hypoxia in the central NP and a more acidic $\mathrm{pH}$ due to accumulation of lactate metabolites [5]. There is an increased production of matrix degrading enzymes, as well as of pro-inflammatory mediators [6], increased cellular 
senescence and enhanced apoptosis of local IVD cells associated with autophagy [7].

The complement is part of the innate immune system and is known to trigger inflammation and subsequent inflammation-mediated tissue damage [8]. The complement system activation has been shown to be abnormally high in human osteoarthritic joints, and the terminal complement complex (TCC) formation has been further identified as crucial for the development of osteoarthritis in mouse stifle joints [9]. Moreover, TCC deposition has been detected in human lumbar spine herniated and degenerated IVD tissues, with predominant presence in AF cells [10].

The complement system can be mainly activated via three different pathways: classical, alternative or lectin [11]. Several triggers of complement activation are also known to play a role in the pathomechanism of disc degeneration, including immunoglobulin deposition [12], specific matrix fragments, such as fibromodulin, aggrecan or fibronectin fragments [13], and cathepsins D and K [14]. It has also been shown that NP cells express toll-like-receptors (TLRs) and that the expression levels of TLR 2 and TLR4 correlate with the degree of disc degeneration [15]. Recent studies indicate that TLRs and the complement system, both part of the innate immunity, are connected via common activators and intracellular regulators $[16,17]$. TCC can be directly regulated by different plasma or membrane-bound inhibitory proteins. Vitronectin and clusterin can be found in the plasma; they bind to C5b-7 and inhibit C5b-9/TCC generation [18]. CD59 is a membrane bound inhibitor that blocks C9 association with $\mathrm{C} 5 \mathrm{~b}-8$ to prevent $\mathrm{C} 5 \mathrm{~b}-9 / \mathrm{TCC}$ deposition on the host cell [18].

TCC deposition on the cell membrane is considered a trigger of inflammation [19] and could therefore, via induction of a pro-inflammatory environment, contribute to disc degeneration-associated matrix degradation as described for other tissues. In human chondrocytes, TCC has been shown to induce up-regulation of MMP1, 3 and 13, ADAMTS4 and 5, CCL5 and COX2 [9]. After in vitro cartilage trauma, enhanced TCC deposition was observed on chondrocytes, together with upregulation of $R U N X 2$ and $V E G F$, important regulators of hypertrophy/senescence, which demonstrated induction of regulated cell death, comprising apoptosis and necroptosis [20]. However, there are no data regarding a TCC-induced pro-inflammatory or catabolic response in IVD cells. Also, the existence and possible pathogenetic implication of inhibitory mechanisms of TCC formation involving CD59 have not been investigated in disc cells or tissues.

Based on these findings, a pathogenetic role of the complement system in the development and progression of disc degeneration can be suggested. We therefore hypothesize that TCC deposition plays a role in the pathomechanism of disc degeneration and that it can be initiated by known inductors of disc degeneration. To study this hypothesis, we have addressed the following research questions:

1. Is TCC deposition higher in tissue samples from patients with various disc pathologies compared to age-matched controls?

2. Is the expression of CD59, a central inhibitory protein of TCC formation, associated with disc pathologies and TCC deposition?

3. Is the formation of TCC and/or expression of CD59 correlated with the age and/or degree of disc degeneration assessed by MRI-based scores?

\section{Methods}

\section{Ethical approval}

Informed consent for sample collection was obtained from each patient, and the study was approved by the local ethical committees (208/15 Ulm University, Germany; S-051/2016 Heidelberg University, Germany; 532-04 Regional Ethical Board Gothenburg, Sweden).

\section{Sample collection}

A total of 49 disc samples were obtained from different patients undergoing surgery in the lumbar region. Open surgery was performed to remove the disc tissue, occasionally including cartilaginous and bony EP (partially or completely, depending on the procedure). All tissues were treated similarly, collected under sterile conditions and directly transferred from the patient to sterile Dulbecco's modified Eagle's medium (Gibco) supplemented with 5\% penicillin-streptomycin $(10,000 \mathrm{U} / \mathrm{mL}-10,000 \mu \mathrm{g} / \mathrm{mL}$, Gibco), 5\% Amphotericin B (250 $\mu \mathrm{g} / \mathrm{mL}$, Biochrom $)$ and $1.5 \% 5 \mathrm{M} \mathrm{NaCl} / 0.4 \mathrm{M} \mathrm{KCl}$ solution (to adjust osmolarity to $400 \mathrm{mOsm}$ ). A total of 10 patients were diagnosed with adolescent idiopathic scoliosis, but no signs of disc degeneration (AIS group, 8 female $/ 2$ male, age $=17 \pm 4$ years old) and 39 with disc degeneration (DD group, 24 female/15 male, age $=63 \pm 12$ years old). For DD patients, Pfirrmann grade and Modic changes were assessed. Control discs were collected post-mortem from a total of 21 donors, which did not reveal significant morphological/histopathological changes. Multilevel sections of the lumbar spine were collected during autopsy (with 24-48 h after death), dissected to remove muscles and ligaments and directly transferred to formalin. The samples were analysed separately considering 2 different age groups: Young ( 6 female $/ 5$ male, age $=7 \pm 7$ years old) and Elder ( 5 female $/ 5$ male, age $65 \pm 15$ years old). Patient information is summarized in Fig. 1a. A detailed donor list is provided in Supplementary Table S1. 
a

\begin{tabular}{lccc}
\hline Condition & $\begin{array}{c}\text { Number of } \\
\text { histological } \\
\text { samples }\end{array}$ & $\begin{array}{c}\text { Age range } \\
\text { (years old) }\end{array}$ & $\begin{array}{c}\text { Mean age } \\
\text { (years old) }\end{array}$ \\
\hline $\begin{array}{l}\text { Post-mortem control donors } \\
\text { - Young }\end{array}$ & 11 & $0-18$ & $7 \pm 7$ \\
- Elder & 10 & $43-83$ & $65 \pm 15$ \\
$\begin{array}{l}\text { Surgery patients } \\
\text { - Adolescent idiopathic } \\
\quad \text { scoliosis (AIS) }\end{array}$ & 10 & $13-25$ & $17 \pm 4$ \\
- Disc degeneration (DD) & 39 & $27-82$ & $63 \pm 12$ \\
\hline
\end{tabular}

b

Age distribution

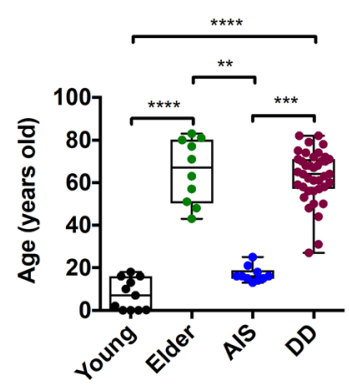

C Age vs Pfirrmann grade in DD

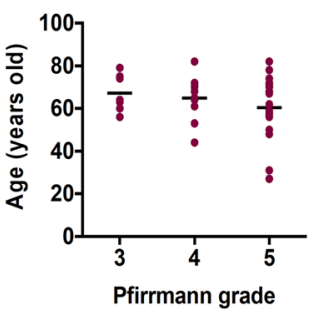

d

Age vs Modic changes in DD

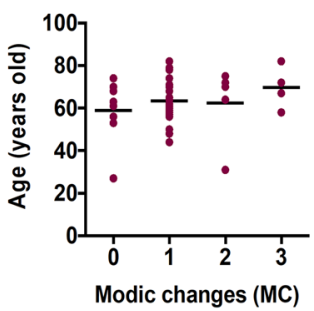

e Modic changes (MC) and
Pfirrmann grade in DD

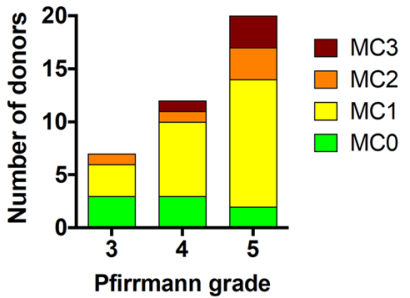

Fig. 1 Donor information (a). IVD tissue donors' distribution through 4 different groups, considering age and pathology: (1) young healthy donors (Young); (2) elder healthy donors (Elder); (3) patients diagnosed with adolescent idiopathic scoliosis and displaying no signs of degeneration (AIS); and (4) patients diagnosed with disc degeneration (DD). Age distribution by donor group (b). Results are displayed in box-and-whisker plots as median \pm interquartile range $(n=10-39$ donors per group). $* * P<0.01 ; * * * P<0.001 ; * * * * P<0.0001$, Kruskal-Wallis test. Age versus Pfirrmann grade (c) and age versus Modic changes (d) in DD patients ( $n=4-22$ donors per group). Results are displayed in $x y$ plots. DD donors' distribution by Modic changes (MC) and Pfirrmann grade ( $n=7-20$ donors per group) (e). Results are shown in a stacked bar plot

\section{Sample processing and histological analysis}

Within a time period of maximum $16 \mathrm{~h}$ after surgery, the disc tissue samples were dissected and separated into AF, NP and EP fragments according to their morphology. Randomly selected fragments from the 3 regions were fixed in $4 \%$ buffered formalin. Tissue fragments containing bone were then decalcified in ethylenediamine tetraacetic acid (EDTA)-glycerol solution before embedding. For the healthy donors, paraffin blocks containing 1-2 complete discs (including EP) were prepared. Tissue sections with $5 \mu \mathrm{m}$ thickness were collected, deparaffinized in xylene solution and rehydrated through a graded series of ethanol. Safranin-O/Fast Green staining was performed to identify proteoglycans distribution.

\section{Immunohistochemistry}

The identification and local distribution of TCC and its inhibitor CD59 were assessed by avidin-biotin complex (Vector laboratories) immunohistochemistry (IHC) using NovaRED Peroxidase (HRP) Substrate Kit (Vector laboratories). Antigen retrieval was performed through incubation with hyaluronidase $(2 \mathrm{mg} / \mathrm{mL}$ in $10 \mathrm{mM}$ citrate buffer, $\mathrm{pH} 8$, $30 \mathrm{~min}, 37^{\circ} \mathrm{C}$ ). Sections were incubated with mouse antihuman C5b-9/TCC (1:250, Quidel) or mouse anti-human CD59 (1:125, Bio-Rad) antibodies, overnight at $4{ }^{\circ} \mathrm{C}$, followed by incubation with goat anti-mouse $\operatorname{IgG}(\mathrm{H}+\mathrm{L})$, Biotin-XX (1:200, Invitrogen) antibody. Isotype controls were also stained with mouse IgG antibody (BioLegend) to prove staining specificity. The stained sections were imaged 

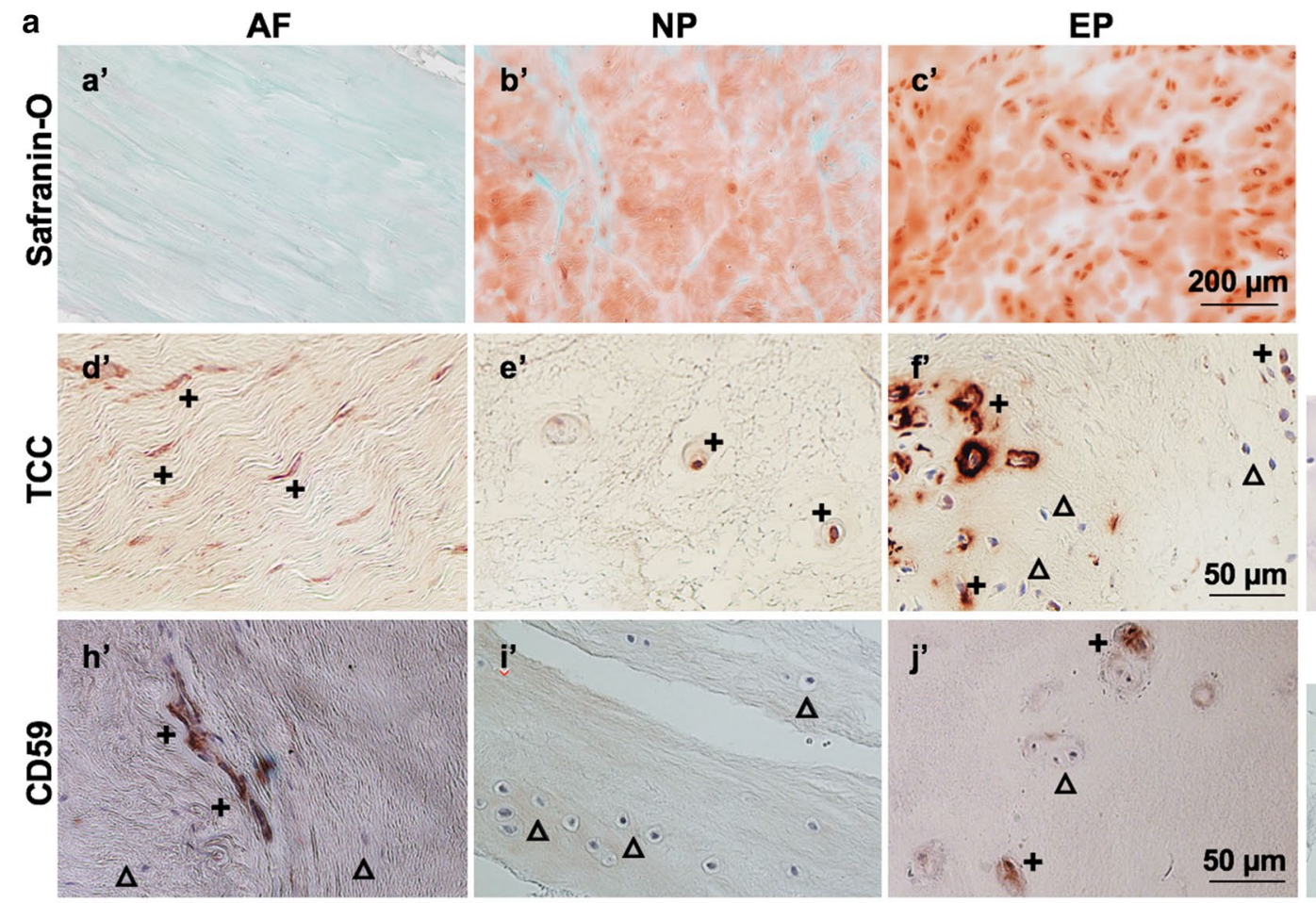

Isotype control
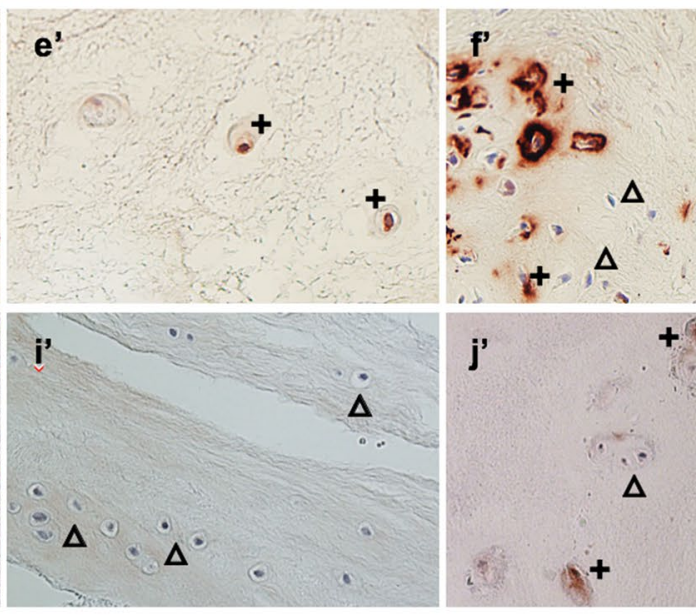

b

Total IVD tissue

AF
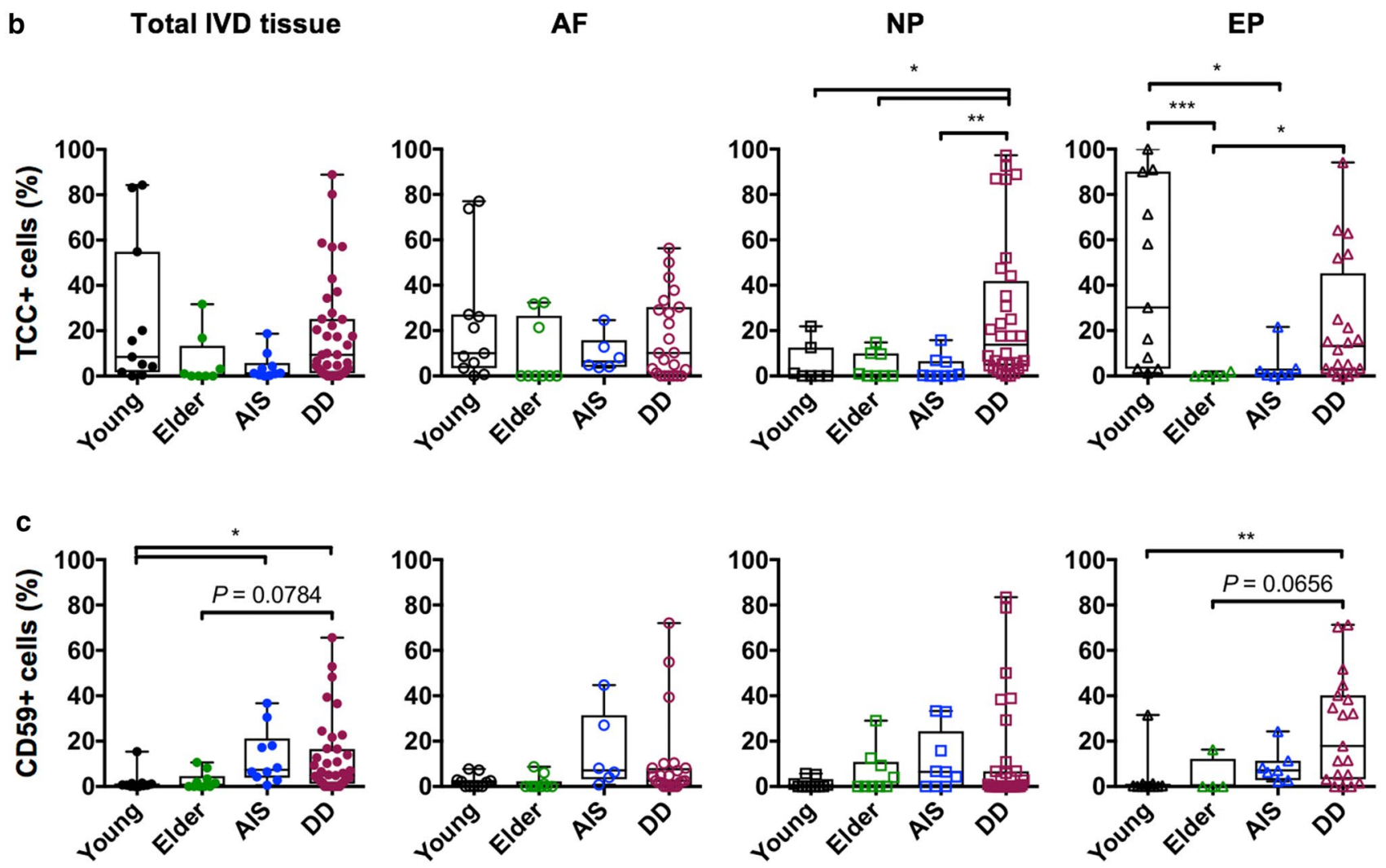

with light microscopy (Zeiss). Positive cells for TCC formation (TCC+) and CD59 expression (CD59+) were manually counted within every region of interest (AF, NP or EP tissue) and normalized by the total cell number within the same region. Results are shown in percentage. Correlation analyses between the percentage of TCC + and CD59+ cells versus donor age, Pfirrmann grade and Modic changes were also performed. 
४Fig. 2 Histopathological analysis of IVD tissue. Representative images of IVD tissue collected during surgery, from a patient diagnosed with disc degeneration (DD), stained for Safranin-O (proteoglycans are stained red, while Fast Green counterstains the non-collagen sites), TCC and CD59, displaying positive (+) and negative $(\Delta)$ cells for each staining, in the different IVD regions: annulus fibrosus (AF), nucleus pulposus (NP) and endplate (EP), and a representative negative isotype control for each immunostaining (a). Percentage of TCC + cells (b) and of CD59+ cells (c) in the total IVD tissue and in $\mathrm{AF}, \mathrm{NP}$ and $\mathrm{EP}$ regions. Tissues were collected post-mortem from Young and Elder donors, or during surgery, from patients diagnosed with adolescent idiopathic scoliosis (AIS) or disc degeneration (DD). Results are presented in box-and-whisker plots as median \pm interquartile range ( $n=4-39$ donors per group). $* P<0.05 ; * * P<0.01$; $* * * P<0.001$, Kruskal-Wallis test

\section{Statistical analysis}

Normality was assessed by D'Agostino-Pearson omnibus normality test, after which statistical analysis was performed with nonparametric Kruskal-Wallis test, followed by Dunn's multiple comparison test. Nonparametric Spearman test was used to analyse possible linear correlations between the percentage of TCC + and CD59+ cells versus donor age, whereas linear regression analysis was used to evaluate possible correlation between the percentage of TCC + and CD59+ cells versus Pfirrmann grade and Modic changes. All tests were performed using GraphPad Prism 7. Statistical significance was considered for $P<0.05$.

\section{Results}

\section{Characterization of human IVD tissue}

To evaluate the complement activation in disc tissues from AIS and DD patients, disc samples from age-matched healthy controls (Young and Elder groups) were used. The average age was similar between Young donors and AIS patients, as well as between Elder and DD (Fig. 1b). Possible correlations between the age of DD donors and Pfirrmann grade (Fig. 1c) or Modic changes (Fig. 1d) were assessed, but no correlation was found. Furthermore, no correlation was found between age, Pfirrmann grade or Modic changes and different DD diagnoses: disc herniation (DH), spondylolisthesis (SL), osteochondrosis (OC) and others including compressive fracture, fall back surgery syndrome, degenerative scoliosis, chronic low back pain and stenosis (Supplementary Fig. S1).

In the tissue biopsies collected from different donors, immunohistochemical evaluations for TCC and CD59 were performed. Furthermore, to better identify the tissue regions (if AF, NP or EP) in fragmented tissue biopsies, samples were stained for Safranin-O/Fast Green (Fig. 2a, $a^{\prime}-c^{\prime}$, representative DD sample). Generally, in the NP and EP, a red staining for proteoglycans was observed, and the staining intensity was consistently stronger in the EP than in the NP. In the NP, an unorganized fiber network containing few cells was found, whereas the EP presented higher cell number and a hyaline cartilage morphology. In contrast, the AF lamellar region exhibited a strong green counterstaining, indicating low proteoglycan content. Elongated AF cells and concentric lamellae fibers were also observed. These known characteristics were considered for the distinction of the different regions in the immunostainings.

Pericellular immunostainings for TCC $\left(\mathrm{d}^{\prime}-\mathrm{f}^{\prime}\right)$ and CD59 $\left(h^{\prime}-j^{\prime}\right)$ are also depicted in Fig. 2a. No immunoreactivity was found in the isotype controls $\left(\mathrm{g}^{\prime}\right.$ and $\left.\mathrm{k}^{\prime}\right)$. The number of positive cells is shown in percentage to the total number of cells identified in delimited regions of AF, NP and EP. In the total IVD tissue analysed and in the AF region, no differences in TCC immunopositivity between the different donor groups were observed (Fig. 2b). However, higher percentage of TCC + cells was found in the NP of DD, when compared to Young $(P<0.05)$, Elder $(P<0.05)$ and AIS $(P<0.01)$ groups. In the EP, higher positivity for TCC was found in Young versus Elder $(P<0.001)$ and AIS $(P<0.05)$, and in DD compared to Elder $(P<0.05)$. TCC immunopositivity was also compared between AF, NP and EP for the different donor groups (Supplementary Fig. S2a), and higher percentage of TCC+ cells was observed in the EP versus NP $(P<0.05)$ of Young donors.

Significantly lower percentage of CD59+ cells was found in the total IVD of Young donors versus AIS and DD $(P<0.05$, Fig. $2 \mathrm{c})$. Although no significant differences were observed in AF and NP, there was higher positivity for CD59 in the EP of DD compared to Young donors $(P<0.01)$. When comparing the percentage of CD59+ cells in AF, NP and EP within each donor group (Supplementary Fig. S2b), a significant increase was observed in the EP versus NP of DD patients $(P<0.05)$. No differences were found in TCC and CD59 positivity in the tissues when the patients were grouped by different DD diagnoses: DH, SL, OC and others (Supplementary Fig. S3).

\section{Correlation analyses}

Possible correlations between the Pfirrmann grade and the percentage of TCC + and CD59+ cells (in the total IVD tissue, as well as in the different regions) were addressed (Fig. 3). Overall, there was a larger variation in the percentage of TCC+ cells in all regions of IVDs from DD patients with higher Pfirrmann grade (Fig. 3a). Even so, a positive correlation was found between the Pfirrmann grade and the percentage of TCC + cells in the total IVD tissue $(P<0.05)$ and in the $\mathrm{AF}$ region $(P<0.05)$, suggesting that the higher the degree of degeneration, the more TCC + cells were found in these tissues. Regarding CD59 expression, also a larger 

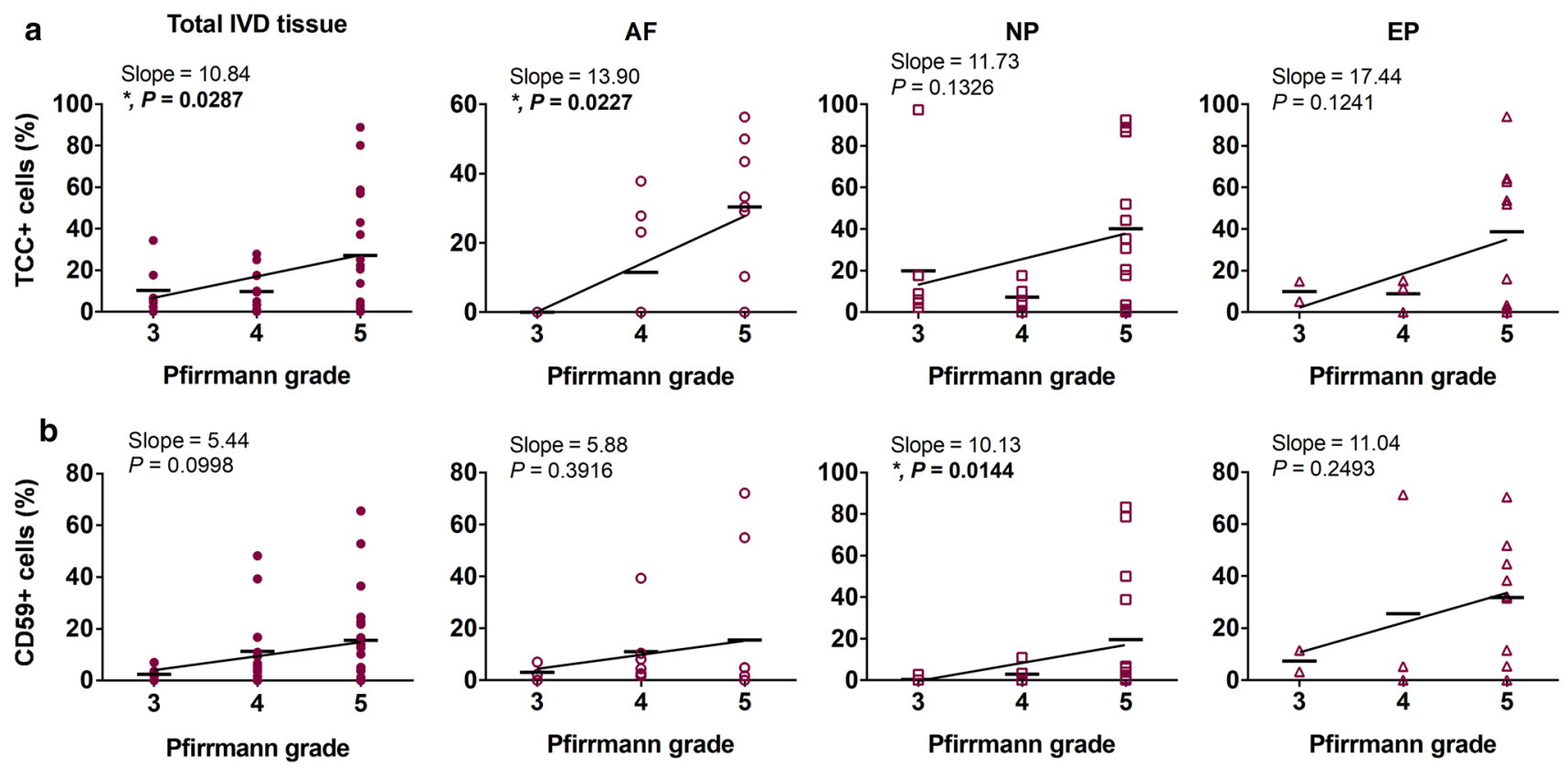

Fig. 3 Linear regression analysis of percentage of TCC+ cells (a) and of CD59+ cells (b) versus Pfirrmann grade in total IVD tissue and in AF, $\mathrm{NP}$ and EP regions for disc degeneration (DD) patients. Results are shown in xy plots ( $n=14-37$ donors)

variation in the percentage of CD59+ cells was seen in all regions of IVDs from DD patients with higher Pfirrmann grade, but no correlation was observed (Fig. 3b).

Possible correlations between Modic changes and the percentage of TCC+ and CD59+ cells in DD tissues were also investigated (Fig. 4). A higher number of DD patients with Modic changes type 1 (MC1) displayed higher percentage of TCC+ and CD59+ cells; however, a correlation pattern was not identified.
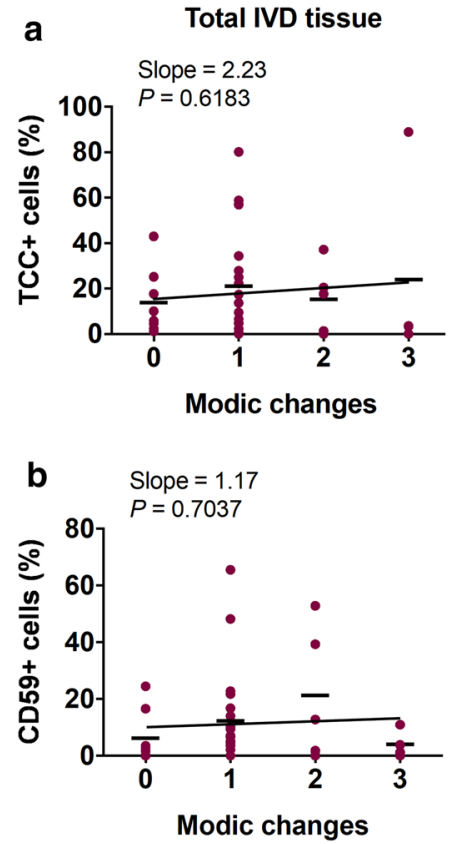
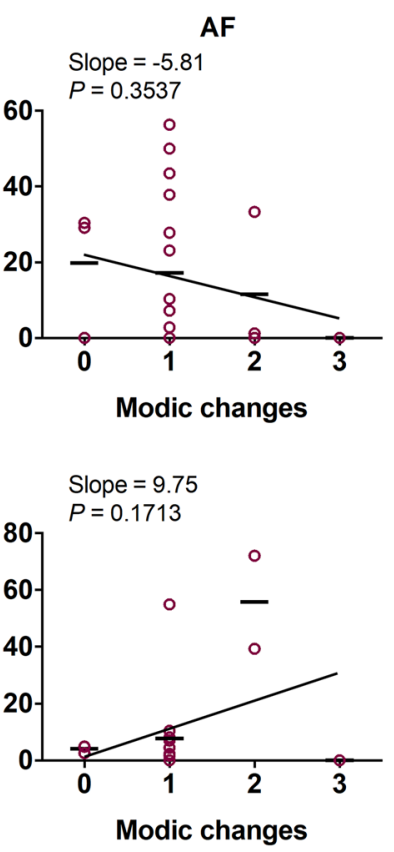
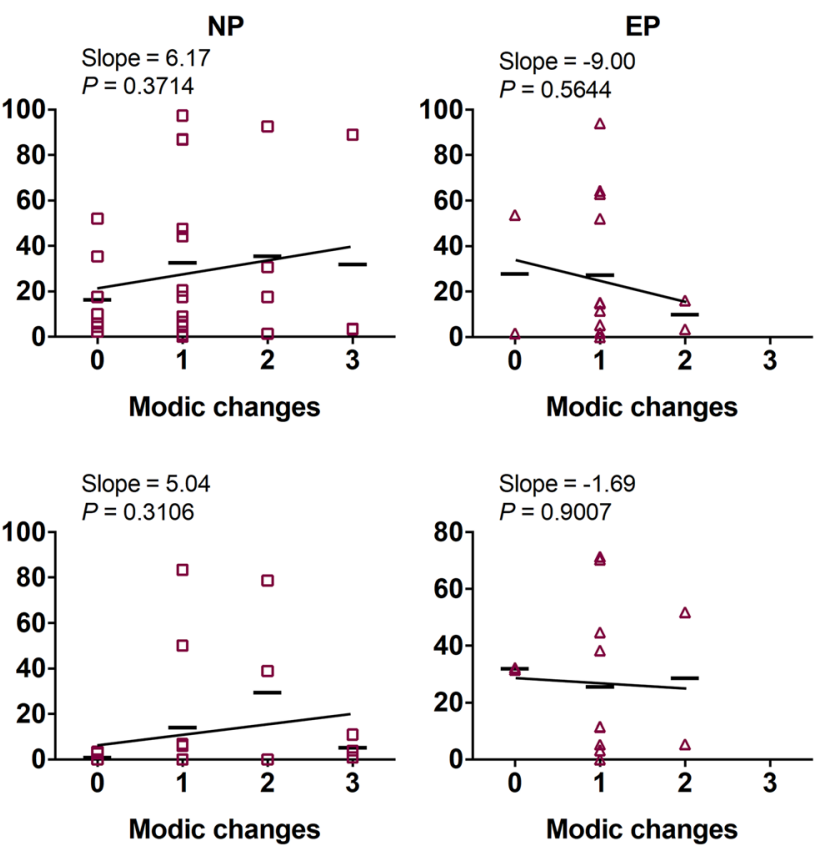

Fig. 4 Linear regression analysis of percentage of TCC+ cells (a) and of CD59+ cells (b) versus Modic changes in total IVD tissue and in AF, $\mathrm{NP}$ and EP regions for disc degeneration (DD) patients. Results are shown in xy plots ( $n=14-37$ donors) 
Correlations between the percentage of $\mathrm{TCC}+$ and CD59+ cells and the age of the patients were also evaluated by linear Spearman correlation analysis. A negative correlation was found between the percentage of TCC + cells and age in the EP of Young donors $(P<0.05$, Table 1), indicating that the high TCC positivity observed in the EP of very young children decreases with time. In contrast, the correlation analysis of percentage of CD59+ cells versus age showed negative correlations in AIS and DD groups for the total IVD tissue $(P<0.05$, Table 2$)$, $\mathrm{AF}(P<0.01)$ and $\mathrm{EP}(P<0.05)$, demonstrating that in the pathologic groups there is a decrease in CD59 expression with age. However, a positive correlation between TCC formation and age for AIS and DD patients was not observed (Table 1).

Table 1 Spearman correlation analysis between percentage of TCC+ cells detected on the total IVD tissue analysed and on the different tissue regions and donors' age $(n=4-32)$

\begin{tabular}{lllll}
\hline Group & Tissue & & & \\
\cline { 2 - 5 } & Total IVD & $\mathrm{AF}$ & $\mathrm{NP}$ & $\mathrm{EP}$ \\
\hline Young & $R=-0.1701$ & $R=-0.1507$ & $R=0.4473$ & $\boldsymbol{R}=-\mathbf{0 . 6 3 9 3}$ \\
& $(P=0.6145)$ & $(P=0.6565)$ & $(P=0.3143)$ & $(*, P=\mathbf{0 . 0 3 8 6})$ \\
Elder & $R=-0.1376$ & $R=0.1683$ & $R=-0.6849$ & $R=0.1309$ \\
& $(P=0.7026)$ & $(P=0.6627)$ & $(P=0.0714)$ & $(P>0.9999)$ \\
AIS & $R=-0.0615$ & $R=0.6179$ & $R=0.3304$ & $R=0.2661$ \\
& $(P=0.8706)$ & $(P=0.2444)$ & $(P=0.3701)$ & $(P=0.5563)$ \\
DD & $R=0.0333$ & $R=-0.1778$ & $R=-0.1479$ & $R=-0.1901$ \\
& $(P=0.8406)$ & $(P=0.4170)$ & $(P=0.4191)$ & $(P=0.4358)$ \\
\hline
\end{tabular}

$I V D$ intervertebral disc, $A F$ annulus fibrosus, $N P$ nucleus pulposus, $E P$ endplate, $A I S$ adolescent idiopathic scoliosis, $D D$ disc degeneration

Bold values indicate statistical significance

\section{Discussion}

DD is more commonly seen in patients with severe low back pain, compared to the general population, and it has been considered as one major pain source, having high clinical relevance. The immune system is known to contribute to DD, but there is limited knowledge regarding a possible involvement of the complement system activation and, especially, TCC formation in the pathomechanism of DD. Disc cells can be directly influenced by immune cells or by the factors they produce [6]; however, this study does not take into consideration other molecules or cells associated with the immune response. This work focused on TCC formation and its regulation by CD59.

TCC has been shown to play a role in articular cartilage disorders, such as osteoarthritis [9] and to be present during disc degeneration [10]; yet, the age-related and spatial profile of TCC deposition in the IVD under different DD phenotypes remains to be defined. Given the similarities between articular cartilage and the IVD, we performed a comprehensive study regarding the identification and localization of TCC and its inhibitor CD59 in AF, NP and EP regions of human disc tissues from DD patients, to learn more about pathomechanisms in DD which could support the work of identifying new therapeutic targets. Therefore, disc biopsies were collected from different patient groups, particularly with different age range and disc pathologies. Possible correlations between TCC and CD59 positivity with donor age, detailed pathology and degree of DD (Pfirrmann grade, Modic changes) were also investigated. As it is difficult to have access to age-related non-pathologic disc tissues, the control groups included disc tissues collected post-mortem from very young (range 0-18 years) or elder (range 43-83 years) donors without known or macroscopically evident disc pathology. The respective age range could
Table 2 Spearman correlation analysis between percentage of CD59+ cells detected on the different tissue regions and donors' age $(n=4-32)$

\begin{tabular}{lllll}
\hline Group & Tissue & & & \\
\cline { 2 - 5 } & Total IVD & $\mathrm{AF}$ & $\mathrm{NP}$ & $\mathrm{EP}$ \\
\hline Young & $R=0.2271$ & $R=0.1728$ & $R=-0.1590$ & $R=-0.2670$ \\
& $(P=0.4960)$ & $(P=0.6069)$ & $(P=0.6423)$ & $(P=0.4193)$ \\
Elder & $R=-0.2780$ & $R=-0.0075$ & $R=-0.1278$ & $R=0.2582$ \\
& $(P=0.4373)$ & $(P>0.9999)$ & $(P=0.7599)$ & $(P>0.9999)$ \\
AIS & $R=-\mathbf{0 . 6 4 6 2}$ & $R=-\mathbf{0 . 7 0 6 2}$ & $R=-0.0261$ & $R=-\mathbf{0 . 8 0 0 1}$ \\
& $(*, P=\mathbf{0 . 0 4 8 1})$ & $(* *, P=\mathbf{0 . 0 0 2 0})$ & $(P=0.9530)$ & $(*, \boldsymbol{P = 0 . 0 4 1 3})$ \\
DD & $R=-\mathbf{0 . 3 8 0 0}$ & $R=-\mathbf{0 . 5 9 7 0}$ & $R=-0.2318$ & $R=-\mathbf{0 . 6 5 3 8}$ \\
& $(*, P=\mathbf{0 . 0 1 7 0})$ & $(* *, P=\mathbf{0 . 0 0 2 6})$ & $(P=0.2017)$ & $(* *$, \\
& & & & $\boldsymbol{P = 0 . 0 0 2 4 )}$ \\
\hline
\end{tabular}

$I V D$ intervertebral disc, $A F$ annulus fibrosus, $N P$ nucleus pulposus, $E P$ endplate, $A I S$ adolescent idiopathic scoliosis, $D D$ disc degeneration

Bold values indicate statistical significance 
be better compared with the two patient groups AIS (range 13-25 years) and DD (range 27-82 years).

In tissue samples from DD patients, a positive correlation was found between TCC deposition in NP and EP with progression of disc degeneration, determined by the MRI-based Pfirrmann grade. Moreover, although with no statically significant differences, a higher number of discs from the DD group displayed MC1, with higher TCC deposition being found in some of these tissues. While $\mathrm{MC} 1$ is associated with inflammation and vascularization of the vertebral body, MC2 is characterized by fatty tissue replacement [21, 22]. The risk to develop Modic changes in the disc has been described to most likely depend on the inflammatory potential of the disc and the capacity of the bone marrow to respond to it [21]. According to the research of Dudli et al. [22], MC1 is associated with the autoimmune response caused by the degenerative milieu in the disc. NP cells are known as immunogenic, but the presence of $\mathrm{MC} 1$ was shown to strongly amplify the immune response [22]. Our results provide further support to the findings from Grönblad and colleagues [10], who reported TCC deposition in disc cells from $36.2 \%$ (21/58) of the patients diagnosed with disc herniation and from $36.4 \%$ (4/11) with DD. Although quantitative comparisons of TCC deposition in different IVD regions was not performed, Grönblad et al. [10] found most TCC deposition in the AF region. While they included in their study mostly patients diagnosed with cauda equina syndrome, spinal stenosis or disc herniations [10], we have included tissues from patients with different phenotypes of disc degeneration-associated diseases (not only disc herniation, but also spondylolisthesis and osteochondrosis, among others), but no differences were found when the results were evaluated separately, depending on the specific DD diagnosis. Experimental studies provided findings from which a potential role of TCC in degeneration and possible new therapeutic options could be concluded. After medial meniscectomy, mice deficient for complement components C5 and C6 display less cartilage degeneration, and lower expression of inflammatory and degradative molecules compared to wild type animals [9]. Reduced ability to form TCC protected the mice towards cartilage degeneration [9].

Regarding non-degenerated controls, TCC deposition was not observed by Grönblad et al. [10] in control tissues from organ donors ( 9 discs, mean age, 51 years; range 39-79 years), which was confirmed in our study for the Elder group, although in single cases TCC + cells could still be detected. Interestingly, in our work, significantly high TCC formation was found in very young donors without obvious disc pathology (under 1 year old), particularly in the EP, which decreased with age. These findings may correlate with the fact that in the earlier stage of life, the EP cartilage is not yet fully developed [23, 24]. A network of microscopic blood vessels infiltrates the EP during spine development, primarily to provide nutrition to the cells, and it only disappears during skeletal maturity [23, 24]. With age and DD progression, calcification of the cartilaginous EP occurs due to occlusion of the marrow spaces, leading to loss of contact between the capillaries and the cartilaginous EP which inhibits the diffusion of solutes from the capillaries to the disc $[25,26]$. Complement is known to be involved in bone disorders, but also in bone homeostasis. TCC components $\mathrm{C} 5$ and $\mathrm{C} 9$ were found to be predominantly expressed in the hypertrophic zone of the growth plate, required for bone formation and ossification [27]. Riegger et al. [20] hypothesized that as TCC induces chondrocytes hypertrophy and apoptosis during endochondral ossification [28], similar mechanisms may lead to degeneration in mature cartilage. In our study, since high TCC deposition was observed in the EP of Young and DD groups, comparable processes seem to occur during IVD development and degeneration.

In AIS patients, similarly to Elder controls, low percentage of TCC+ cells was found in the different tissues, which might indicate that, although strong TCC deposition may be a degeneration-associated event, cells in non-degenerated discs are not completely protected towards TCC formation at sublytic concentrations. Complement proteins have been identified in endochondral bone formation and are described to be involved in cartilage cell death and vascularization [27]. This may be especially relevant during the early stages of skeletal development and, later, partly persist on a low level. This mechanism might be compensated during ageing, but possibly increases with DD.

Additionally, CD59 expression shared a similar pattern to TCC in degenerated discs, particularly in the EP. CD59 is known to regulate bone growth and homeostasis [28, 29], and to play a role in arthritis in human [29, 30]. Moreover, after medial meniscectomy, mice genetically deficient for CD59a displayed higher cartilage degradation than wild type [9]. In our study, negative correlations were observed between the percentage of CD59+ cells and the age of the patients in AIS and DD, indicating that CD59 expression is decreasing with age in the AF and EP of pathologic discs, which might contribute to an increased cell death described in the natural aging process of the IVD [3]. Nonetheless, a direct correlation between TCC formation and age for AIS and DD patients was not observed, indicating that other molecules than CD59 may play a role in TCC regulation in the IVD. Moreover, although some disc tissues from DD patients displayed immunopositivity for CD59, this protective mechanism towards TCC formation seems to be less effective in elder patients. Literature findings indicate that complement activation can increase the expression of CD59 in membranous glomerulonephritis [31], colorectal cancer [32], degenerated cartilage [20] and several other degenerative diseases which can be interpreted as a protective mechanism [31, 32]. However, this protective mechanism may not be activated in the IVD since no significant increase of TCC formation was observed with aging. 
Rajasekaran et al. [33] have recently observed an upregulation of complement proteins (such as haptoglobin, complement $\mathrm{C} 3, \mathrm{C} 5$ and factor $\mathrm{H}$, among others) in discs from $\mathrm{DD}$ patients (5 discs, age range 34-72 years old donors) compared to healthy discs (3 discs, age range 13-32 years old donors). Nonetheless, except Grönblad et al. [10], no further studies have investigated, so far, the presence and relevance of TCC formation in disc tissue from patients with different disc degenerative diseases. The findings of our immunohistochemical investigations suggest a possible role of TCC in disc development, as well as in degeneration. Our results further indicate that there is a balance between TCC deposition and CD59 expression, and that CD59 expression follows TCC deposition in DD— to protect the cells towards the lytic or sublytic activity of TCC, which could explain why with progression of degeneration both higher TCC deposition and CD59 expression were found in some DD tissues.

\section{Conclusion}

In conclusion, our findings indicate that the complement system and specifically TCC deposition might be associated with DD. Further studies are ongoing to better understand which local microenvironmental factors can activate TCC formation, the effect of sublytic concentrations of TCC in disc cells and the contribution to cell death. These studies should indicate if TCC can be a target for therapeutic intervention in DD.

Acknowledgements The authors of the present study wish to acknowledge Prof. Thomas Barth, Institute of Pathology at Ulm University, for providing paraffin sections of healthy intervertebral discs, Prof. Benjamin Mayer, Biometrics Department at Ulm University, for the support with the statistical analysis, Mrs. Iris Baum and Mrs. Marion Tomo, Dr. Helga Joos and Dr. Taryn Saggese for their technical support.

Funding Open Access funding enabled and organized by Projekt DEAL. This study was supported by the German Research Foundation (NE 549/6-1, BR 919/12-1), Ulm University (L.SBN.0157), German Academic Exchange Service, Conselho de Reitores das Universidades Portuguesas and the Alexander von Humboldt Foundation. Open Access funding enabled and organized by Projekt DEAL.

\section{Compliance with ethical standards}

Conflict of interest The authors declare that they have no conflict of interest.

Ethical approval Informed consent for sample collection was obtained from all individual patients and the study was approved by the local ethical committees (208/15 Ulm University, Germany; S-051/2016 Heidelberg University, Germany; 532-04 Regional Ethical Board Gothenburg, Sweden).

Open Access This article is licensed under a Creative Commons Attribution 4.0 International License, which permits use, sharing, adaptation, distribution and reproduction in any medium or format, as long as you give appropriate credit to the original author(s) and the source, provide a link to the Creative Commons licence, and indicate if changes were made. The images or other third party material in this article are included in the article's Creative Commons licence, unless indicated otherwise in a credit line to the material. If material is not included in the article's Creative Commons licence and your intended use is not permitted by statutory regulation or exceeds the permitted use, you will need to obtain permission directly from the copyright holder. To view a copy of this licence, visit http://creativecommons.org/licenses/by/4.0/.

\section{References}

1. Vos T, Flaxman AD, Naghavi M, Lozano R, Michaud C, Ezzati $\mathrm{M}$ et al (2012) Years lived with disability (YLDs) for 1160 sequelae of 289 diseases and injuries 1990-2010: a systematic analysis for the Global Burden of Disease Study 2010. Lancet 380:2163-2196. https://doi.org/10.1016/S0140-6736(12)61729 $-2$

2. Battie MC, Videman T, Levalahti E, Gill K, Kaprio J (2007) Heritability of low back pain and the role of disc degeneration. Pain 131:272-280. https://doi.org/10.1016/j.pain.2007.01.010

3. Buckwalter JA (1995) Aging and degeneration of the human intervertebral disc. Spine (Phila Pa 1976) 20:1307-1314. https ://doi.org/10.1097/00007632-199506000-00022

4. Adams MA, Roughley PJ (2006) What is intervertebral disc degeneration, and what causes it? Spine (Phila Pa 1976) 31:2151-2161. https://doi.org/10.1097/01.brs.0000231761 $.73859 .2 \mathrm{c}$

5. Urban JP, Smith S, Fairbank JC (2004) Nutrition of the intervertebral disc. Spine (Phila Pa 1976) 29:2700-2709. https://doi. org/10.1097/01.brs.0000146499.97948.52

6. Risbud MV, Shapiro IM (2014) Role of cytokines in intervertebral disc degeneration: pain and disc content. Nat Rev Rheumatol 10:44-56. https://doi.org/10.1038/nrrheum.2013.160

7. Nerlich AG, Weiler C, Zipperer J, Narozny M, Boos N (2002) Immunolocalization of phagocytic cells in normal and degenerated intervertebral discs. Spine (Phila Pa 1976) 27:2484-2490. https://doi.org/10.1097/00007632-200211150-00012

8. Markiewski MM, Lambris JD (2007) The role of complement in inflammatory diseases from behind the scenes into the spotlight. Am J Pathol 171:715-727. https://doi.org/10.2353/ajpat h.2007.070166

9. Wang Q, Rozelle AL, Lepus CM, Scanzello CR, Song JJ, Larsen DM et al (2011) Identification of a central role for complement in osteoarthritis. Nat Med 17:1674-1679. https://doi.org/10.1038/ $\mathrm{nm} .2543$

10. Grönblad M, Habtemariam A, Virri J, Seitsalo S, Vanharanta H, Guyer RD (2003) Complement membrane attack complexes in pathologic disc tissues. Spine (Phila Pa 1976) 28:114-118. https ://doi.org/10.1097/00007632-200301150-00004

11. Ricklin D, Lambris JD (2013) Complement in immune and inflammatory disorders: pathophysiological mechanisms. J Immunol 190:3831-3838. https://doi.org/10.4049/jimmunol.1203487

12. Habtemariam A, Gronblad M, Virri J, Seitsalo S, Ruuskanen M, Karaharju E (1996) Immunocytochemical localization of immunoglobulins in disc herniations. Spine (Phila Pa 1976) 21:18641869. https://doi.org/10.1097/00007632-199608150-00005

13. Happonen KE, Heinegård D, Saxne T, Blom AM (2012) Interactions of the complement system with molecules of extracellular matrix: relevance for joint diseases. Immunobiology 217:10881096. https://doi.org/10.1016/j.imbio.2012.07.013

14. Ariga K, Yonenobu K, Nakase T, Kaneko M, Okuda S, Uchiyama $Y$ et al (2001) Localization of cathepsins D, K, and L in degenerated human intervertebral discs. Spine (Phila Pa 1976) 26:26662672. https://doi.org/10.1097/00007632-200112150-00007 
15. Klawitter M, Hakozaki M, Kobayashi H, Krupkova O, Quero L, Ospelt C et al (2014) Expression and regulation of toll-like receptors (TLRs) in human intervertebral disc cells. Eur Spine J 23:1878-1891. https://doi.org/10.1007/s00586-014-3442-4

16. Barratt-Due A, Pischke SE, Nilsson PH, Espevik T, Mollnes TE (2017) Dual inhibition of complement and Toll-like receptors as a novel approach to treat inflammatory diseases-C 3 or C5 emerge together with CD14 as promising targets. J Leukoc Biol 101:193204. https://doi.org/10.1189/jlb.3VMR0316-132R

17. Hajishengallis G, Lambris JD (2010) Crosstalk pathways between Toll-like receptors and the complement system. Trends Immunol 31:154-163. https://doi.org/10.1016/j.it.2010.01.002

18. Noris M, Remuzzi G (2013) Overview of complement activation and regulation. Semin Nephrol 33:479-492. https://doi. org/10.1016/j.semnephrol.2013.08.001

19. Morgan BP (2016) The membrane attack complex as an inflammatory trigger. Immunobiology 221:747-751. https://doi. org/10.1016/j.imbio.2015.04.006

20. Riegger J, Huber-Lang M, Brenner RE (2020) Crucial role of the terminal complement complex in chondrocyte death and hypertrophy after cartilage trauma. Osteoarthr Cartil. https://doi. org/10.1016/j.joca.2020.01.004

21. Dudli S, Fields AJ, Samartzis D, Karppinen J, Lotz JC (2016) Pathobiology of Modic changes. Eur Spine J 25:3723-3734. https ://doi.org/10.1007/s00586-016-4459-7

22. Dudli S, Liebenberg E, Magnitsky S, Lu B, Lauricella M, Lotz JC (2018) Modic type 1 change is an autoimmune response that requires a proinflammatory milieu provided by the 'Modic disc'. Spine J 18:831-844. https://doi.org/10.1016/j.spinee.2017.12.004

23. Moore RJ (2006) The vertebral endplate: disc degeneration, disc regeneration. Eur Spine J 15:S333-S337. https://doi.org/10.1007/ s00586-006-0170-4

24. Taylor JR (1975) Growth of human intervertebral discs and vertebral bodies. J Anat 120:49-68

25. Roberts S, Urban JP, Evans H, Eisenstein SM (1996) Transport properties of the human cartilage endplate in relation to its composition and calcification. Spine (Phila Pa 1976) 21:415-420. https://doi.org/10.1097/00007632-199602150-00003

26. Benneker LM, Heini PF, Alini M, Anderson SE, Ito K (2005) 2004 Young Investigator Award Winner: vertebral endplate marrow contact channel occlusions and intervertebral disc degeneration. Spine (Phila Pa 1976) 30:167-173. https://doi.org/10.1097/01. brs.0000150833.93248.09

27. Andrades JA, Nimni ME, Becerra J, Eisenstein R, Davis M, Sorgente N (1996) Complement proteins are present in developing endochondral bone and may mediate cartilage cell death and vascularization. Exp Cell Res 227:208-213. https://doi.org/10.1006/ excr.1996.0269

28. Bloom AC, Collins FL, Van't Hof RJ, Ryan ES, Jones E, Hughes TR et al (2016) Deletion of the membrane complement inhibitor CD59a drives age and gender-dependent alterations to bone phenotype in mice. Bone 84:253-261. https://doi.org/10.1016/j. bone.2015.12.014

29. Konttinen YT, Ceponis A, Meri S, Vuorikoski A, Kortekangas P, Sorsa $T$ et al (1996) Complement in acute and chronic arthritides: assessment of C3c, C9, and protectin (CD59) in synovial membrane. Ann Rheum Dis 55:888-894. https://doi.org/10.1136/ $\operatorname{ard} .55 .12 .888$

30. Jones J, Laffafian I, Lawson T, Williams BD, Morgan BP (1996) Signalling through neutrophil Fc gamma RIII, Fc gamma RII, and CD59 is not impaired in active rheumatoid arthritis. Ann Rheum Dis 55:294-297. https://doi.org/10.1136/ard.55.5.294

31. Lehto T, Honkanen E, Teppo AM, Meri S (1995) Urinary excretion of protectin (CD59), complement SC5b-9 and cytokines in membranous glomerulonephritis. Kidney Int 47:1403-1411. https ://doi.org/10.1038/ki.1995.197

32. Watson NF, Durrant LG, Madjd Z, Ellis IO, Scholefield JH, Spendlove I (2006) Expression of the membrane complement regulatory protein CD59 (protectin) is associated with reduced survival in colorectal cancer patients. Cancer Immunol Immunother 55:973-980. https://doi.org/10.1007/s00262-005-0055-0

33. Rajasekaran S, Tangavel C, Soundararajan DCR, Nayagam SM, Matchado MS et al (2020) Inflammaging determines health and disease in lumbar discs-evidence from differing proteomic signatures of healthy, aging, and degenerating discs. Spine J 20:48-59. https://doi.org/10.1016/j.spinee.2019.04.023

Publisher's Note Springer Nature remains neutral with regard to jurisdictional claims in published maps and institutional affiliations.

\section{Affiliations}

\section{Graciosa Q. Teixeira ${ }^{1}$ (1) $\cdot$ Zhiyao Yong $^{1} \cdot$ Raquel M. Goncalves ${ }^{1,2,3,4} \cdot$ Amelie Kuhn $^{5} \cdot$ Jana Riegger $^{5} \cdot$ Helena Brisby $^{6,7}$. Helena Barreto Henriksson ${ }^{6,7,8}$. Michael Ruf ${ }^{9} \cdot$ Andreas Nerlich $^{10}$. Uwe M. Mauer ${ }^{11}$ • Anita Ignatius ${ }^{1}$. Rolf E. Brenner ${ }^{5}$. Cornelia Neidlinger-Wilke ${ }^{1}$ (D)}

1 Institute of Orthopaedic Research and Biomechanics, Trauma Research Centre, Ulm University, Ulm, Germany

2 Instituto de Investigação e Inovação em Saúde (i3S), Universidade do Porto, Porto, Portugal

3 Instituto de Engenharia Biomédica (INEB), Universidade do Porto, Porto, Portugal

4 Instituto de Ciências Biomédicas Abel Salazar (ICBAS), Universidade do Porto, Porto, Portugal

5 Division for Biochemistry of Joint and Connective Tissue Diseases, Department of Orthopedics, Ulm University, Ulm, Germany

6 Department of Orthopedics, Institute of Clinical Sciences, Sahlgrenska Academy, Gothenburg University, Gothenburg, Sweden
7 Department of Orthopedics, Sahlgrenska University Hospital, Gothenburg, Sweden

8 Present Address: Department of Clinical Immunology and Transfusion Medicine, Sahlgrenska University Hospital, Gothenburg, Sweden

9 Center for Spine Surgery, Orthopedics, and Traumatology, SRH-Klinikum Karlsbad-Langensteinbach, Karlsbad, Germany

10 Department of Pathology, Academic Hospital Munich-Bogenhausen, Munich, Germany

11 Department of Neurosurgery, German Armed Forces Hospital, Ulm, Germany 